\title{
Physics Potential of a 2540 Km Baseline Superbeam Experiment
}

\author{
Aniket Joglekar*, Suprabh Prakash, Sushant K. Raut † and S. Uma Sankar \\ Department of Physics, Indian Institute of Technology Bombay, Mumbai 400076, India
}

(Dated: November 28, 2018)

\begin{abstract}
We study the physics potential of a neutrino superbeam experiment with a $2540 \mathrm{~km}$ baseline. We assume a neutrino beam similar to the NuMI beam in medium energy configuration. We consider a 100 kton totally active scintillator detector at a $7 \mathrm{mr}$ off-axis location. We find that such a configuration has outstanding hierarchy discriminating capability. In conjunction with the data from the present reactor neutrino experiments, it can determine the neutrino mass hierarchy at $3 \sigma$ level in less than 5 years, if $\sin ^{2} 2 \theta_{13} \geq 0.01$, running in the neutrino mode alone. As a stand alone experiment, with a 5 year neutrino run and a 5 year anti-neutrino run, it can determine non-zero $\theta_{13}$ at $3 \sigma$ level if $\sin ^{2} 2 \theta_{13} \geq 7 \times 10^{-3}$ and hierarchy at $3 \sigma$ level if $\sin ^{2} 2 \theta_{13} \geq 8 \times 10^{-3}$. This data can also distinguish $\delta_{C P}=\pi / 2$ from the CP conserving values of 0 and $\pi$, for $\sin ^{2} 2 \theta_{13} \geq 0.02$.

PACS numbers: 14.60.Pq,14.60.Lm,13.15.+g
\end{abstract}

Keywords:

\footnotetext{
* Address after August 1, 2010: Department of Physics, University of Chicago, Chicago, IL, USA

${ }^{\dagger}$ Email Address: sushant@phy.iitb.ac.in
} 


\section{INTRODUCTION}

During the past decade, great progress has been made in the study of neutrinos. In particular, the mass-squared differences and two of the mixing angles have been measured with good precision. Despite this progress, the following properties of neutrinos are still unknown

- The absolute mass scale of the neutrinos,

- Dirac vs Majorana nature of neutrinos,

- The value of the mixing angle $\theta_{13}$,

- Mass pattern or mass hierarchy of the neutrinos,

- $\mathrm{CP}$ violation in neutrino sector and the value of the CP-violating phase $\delta_{C P}$,

- Deviation of $\theta_{23}$ from $\pi / 4$.

KATRIN tritium decay experiment [1, 2] and various neutrinoless double beta decay experiments [3] currently underway will address the first two problems. The next four problems fall under the purview of neutrino oscillations and hence are expected to be solved by the current and future neutrino oscillation experiments. There are three efforts underway to determine the mixing angle $\theta_{13}$ using reactor neutrino data. They are (a) Double Chooz,

expected to start taking data this year [4] with sensitivity $\sin ^{2} 2 \theta_{13} \geq 0.04$, (b) RENO [5] with sensitivity $\sin ^{2} 2 \theta_{13} \geq 0.02$, and (c) Daya Bay [6] with sensitivity $\sin ^{2} 2 \theta_{13} \geq 0.01$. In addition, there are two accelerator experiments, $\mathrm{T} 2 \mathrm{~K}$ and $\mathrm{NO} \nu \mathrm{A}$, which can determine non-zero $\theta_{13}$. Their sensitivities are $\sin ^{2} 2 \theta_{13} \geq 0.01$ at $90 \%$ confidence level for T2K [7] and $\sin ^{2} 2 \theta_{13} \geq 0.03$ at $3 \sigma$ for $\mathrm{NO} \nu \mathrm{A}$ [8]. Thus it is hoped that the determination of $\theta_{13}$ can be achieved by these experiments within the next decade.

However, it was shown recently [9] that the currently running or planned experiments will be able to determine the mass hierarchy or $\delta_{C P}$ only for very favourable values of $\theta_{13}$ and $\delta_{C P}$. The effect of mass hierarchy and $\delta_{C P}$ are closely interlinked [10 14]. Disentangling these two effects requires considerable effort and possibly data from many experiments [15, 16]. Thus the question of designing a neutrino beam-detector configuration, which will enable us to determine hierarchy and $\delta_{C P}$ unambiguously, acquires great importance. 
There have been numerous proposals made to achieve the above purpose. These include megaton size detectors [17-19], a pair of detectors with different baselines but with the same value of $L / E$ [15, 16] and detectors at the 'magic' baseline of $7500 \mathrm{~km} \mathrm{[20,} \mathrm{21].} \mathrm{Recently}$ we made a proposal of a shorter magical baseline [22], which has exceptional capability to determine mass hierarchy, independently of $\delta_{C P}$. In this report, we study the full physics potential of our proposal.

\section{CALCULATION}

As mentioned before, the unknowns to be determined from the neutrino oscillation experiments are the mixing angle $\theta_{13}$, the neutrino mass hierarchy, the $\mathrm{CP}$ violating phase $\delta_{C P}$ and the octant of $\theta_{23}$. The $\nu_{\mu} \rightarrow \nu_{e}$ oscillation probability $\mathrm{P}_{\mu \mathrm{e}}$ is sensitive to all these unknowns. In this paper, we will not consider the problem of octant ambiguity of $\theta_{23}$. We will assume the true value of $\theta_{23}$ to be its best fit value $\pi / 4$. The expression for $\mathrm{P}_{\mu \mathrm{e}}$, in three flavour oscillations including matter effects, is given as [23-25]

$$
\begin{aligned}
\mathrm{P}_{\mu \mathrm{e}} & =C_{0} \frac{\sin ^{2}((1-\hat{A}) \Delta)}{(1-\hat{A})^{2}} \\
& +\alpha C_{1} \frac{\sin ((1-\hat{A}) \Delta)}{(1-\hat{A})} \frac{\sin (\hat{A} \Delta)}{\hat{A}} \\
& +\alpha^{2} C_{2} \frac{\sin ^{2}(\hat{A} \Delta)}{\hat{A}^{2}}
\end{aligned}
$$

where $\Delta=\left(1.27 \Delta_{31} L / E\right), \hat{A}=A / \Delta_{31}$ and $\alpha=\Delta_{21} / \Delta_{31} \approx \pm 0.04$, as given by the solar and atmospheric neutrino data [26]. Here $L$ is in $\mathrm{km}, E$ is in $\mathrm{GeV}$ and $\Delta_{31}$ is given in units of $\mathrm{eV}^{2}$. The coefficients, $C_{i}$ are given by

$$
\begin{aligned}
& C_{0}=\sin ^{2} \theta_{23} \sin ^{2} 2 \theta_{13} \\
& C_{1}=\cos \theta_{13} \sin 2 \theta_{12} \sin 2 \theta_{13} \sin 2 \theta_{23} \cos \left(\Delta+\delta_{C P}\right) \\
& C_{2}=\sin ^{2} 2 \theta_{12} \cos ^{2} \theta_{23} .
\end{aligned}
$$

The matter effects are given by the matter term [27] $A\left(\right.$ in $\left.\mathrm{eV}^{2}\right)=0.76 \times$ $10^{-4} \rho(\mathrm{gm} / \mathrm{cc}) E(\mathrm{GeV}) \mathrm{eV}^{2} . \quad \rho$ is the density of the matter through which the neutrino propagates. $\Delta_{31}$ and hence $\Delta$ are positive for normal hierarchy $(\mathrm{NH})$ and negative for inverted hierarchy $(\mathrm{IH})$, for both neutrinos and anti-neutrinos. $A$, on the other hand, is 
positive for neutrinos and negative for anti-neutrinos. Therefore, $\hat{A}$ is positive for NH and negative for $\mathrm{IH}$ in the case of neutrinos, and vice versa for anti-neutrinos.

The dependence of $\mathrm{P}_{\mu \mathrm{e}}$ on $\delta_{C P}$ and $\theta_{13}$ is seen explicitly in Eq. (11). In addition, the quantities $\hat{A}$ and $\Delta$ are sensitive to the neutrino mass hierarchy. Thus, this channel which is sensitive to all the three unknowns mentioned above, is a good candidate to probe these unknown parameters. From Eq. (11) we see that $\mathrm{P}_{\mu \mathrm{e}}$ is large for $\Delta=\left(1.27 \Delta_{31} L / E\right) \approx \pi / 2$. On the other hand, the matter term $\hat{A}$ becomes significant at large energies. Therefore, the matter term starts to play an important role in shaping the oscillation probabilities only for long baselines. For a baseline of $732 \mathrm{~km}$, and for fixed values of $\theta_{13}$ and $\delta_{C P}$, it increases $\mathrm{P}_{\mu \mathrm{e}}$ by about $25 \%$ for $\mathrm{NH}$ and decreases it by the same amount for $\mathrm{IH}$ [28]. For longer baselines, the change in the probability is even larger. At shorter baselines, the sensitivity of $\mathrm{P}_{\mu \mathrm{e}}$ to the matter term and hence the mass hierarchy, is low.

Since $\mathrm{P}_{\mu \mathrm{e}}$ depends on three unknowns, $\theta_{13}$, mass hierarchy and $\delta_{C P}$, it is impossible to determine any one of them by making a single measurement. Even if one measures $\mathrm{P}_{\mu \mathrm{e}}$ as a function of energy, it is possible to get degenerate solutions [10 14]. For example, it is possible to have $\mathrm{P}_{\mu \mathrm{e}}\left(\theta_{13}, N H ; E\right)=\mathrm{P}_{\mu \mathrm{e}}\left(\theta_{13}^{\prime}, I H ; E\right)$, as illustrated in Fig 1. Such a degeneracy can be broken, if the value of $\theta_{13}$ can be determined from different experiments. Reactor anti-neutrino experiments with $L \approx 1 \mathrm{~km}$, can determine $\theta_{13}$ unambiguously. The currently planned experiments can measure non-zero values as small as $\sin ^{2} 2 \theta_{13} \geq 0.01$ [4 6]. Thus we expect that this degeneracy can be lifted by the results of reactor neutrino experiments.

Even if the ambiguity due to the value of $\theta_{13}$ is lifted, there is a further ambiguity between hierarchy and $\delta_{C P}$. This ambiguity arises due to the following reason. If we replace $\nu \rightarrow \bar{\nu}$, we have $\delta_{C P} \rightarrow-\delta_{C P}$ and $\hat{A} \rightarrow-\hat{A}$. However, if we flip the hierarchy, we have $\hat{A} \rightarrow-\hat{A}$ and $\Delta \rightarrow-\Delta$. Hence, the change in $\mathrm{P}_{\mu \mathrm{e}}$ due to hierarchy flip can be compensated by changing $\delta_{C P}$. This ambiguity is particularly important for $\sin ^{2} 2 \theta_{13} \leq 0.02$. Therefore, even if we know the value of $\theta_{13}$, it is possible to get degenerate solutions of the kind $\mathrm{P}_{\mu \mathrm{e}}\left(N H, \delta_{C P} ; E\right)=\mathrm{P}_{\mu \mathrm{e}}\left(I H, \delta_{C P}^{\prime} ; E\right)$ over a wide range of energies, as illustrated in Fig. 2, We are led to consider various improvisations to overcome this problem.

In Eq. (1), only the $C_{1}$ term contains $\delta_{C P}$. The hierarchy- $\delta_{C P}$ ambiguity can be lifted if the term containing $C_{1}$ can be chosen to be zero. One simple way to do it is to choose $\hat{A} \Delta=\pi[29]$. This condition can be achieved for both NH and IH simultaneously and gives us 


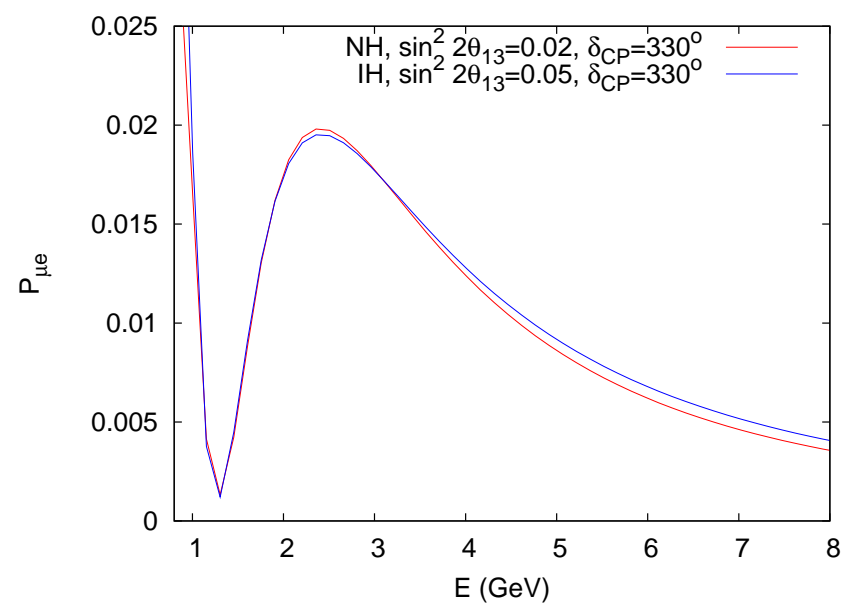

FIG. 1: Hierarchy- $\theta_{13}$ degeneracy in $\mathrm{P}_{\mu \mathrm{e}}$ at $1300 \mathrm{~km}$. We see that $\mathrm{P}_{\mu \mathrm{e}}\left(N H, \sin ^{2} 2 \theta_{13}=0.02 ; E\right)=$ $\mathrm{P}_{\mu \mathrm{e}}\left(I H, \sin ^{2} 2 \theta_{13}=0.05 ; E\right)$. The value of $\delta_{C P}$ is taken to be $330^{\circ}$ here.

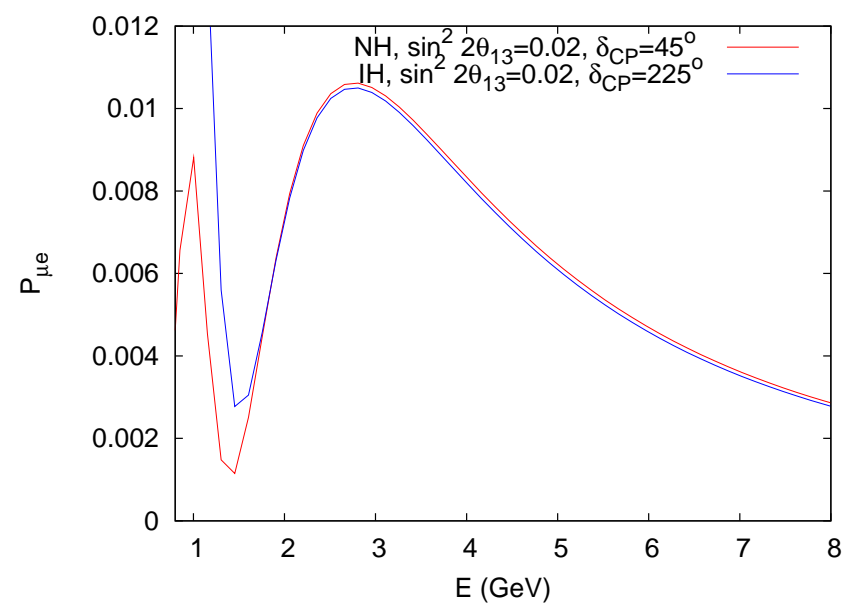

FIG. 2: Hierarchy- $\delta_{C P}$ degeneracy in $\mathrm{P}_{\mu \mathrm{e}}$ at $1300 \mathrm{~km}$. We see that $\mathrm{P}_{\mu \mathrm{e}}\left(N H, \delta_{C P}=45^{\circ} ; E\right)=\mathrm{P}_{\mu \mathrm{e}}\left(I H, \delta_{C P}=\right.$ $\left.225^{\circ} ; E\right)$ for $E>1.5 \mathrm{GeV}$. The value of $\sin ^{2} 2 \theta_{13}$ is taken to be 0.02 here.

an energy independent condition $L \approx 7500 \mathrm{~km}$. This is the famous magic baseline condition and it has been extensively studied [20, 21]. In [22] it was noted that the $C_{1}$ term can be made zero for IH only by choosing $(1-\hat{A}) \Delta=-\pi$. This makes $\mathrm{P}_{\mu \mathrm{e}}(I H)$ independent of $\delta_{C P}$ and also very small $\left(\leq \alpha^{2} \approx 0.002\right)$. If we choose $(1-\hat{A}) \Delta=\pi / 2$ for $\mathrm{NH}$, then $\mathrm{P}_{\mu \mathrm{e}}(N H)$ will be quite siginificant for all values of $\delta_{C P}$. Solving the above pair of simultaneous equations, we obtain $L=2540 \mathrm{~km}$ and $E=3.3 \mathrm{GeV}$. For this baseline, at this energy, there is a clear 


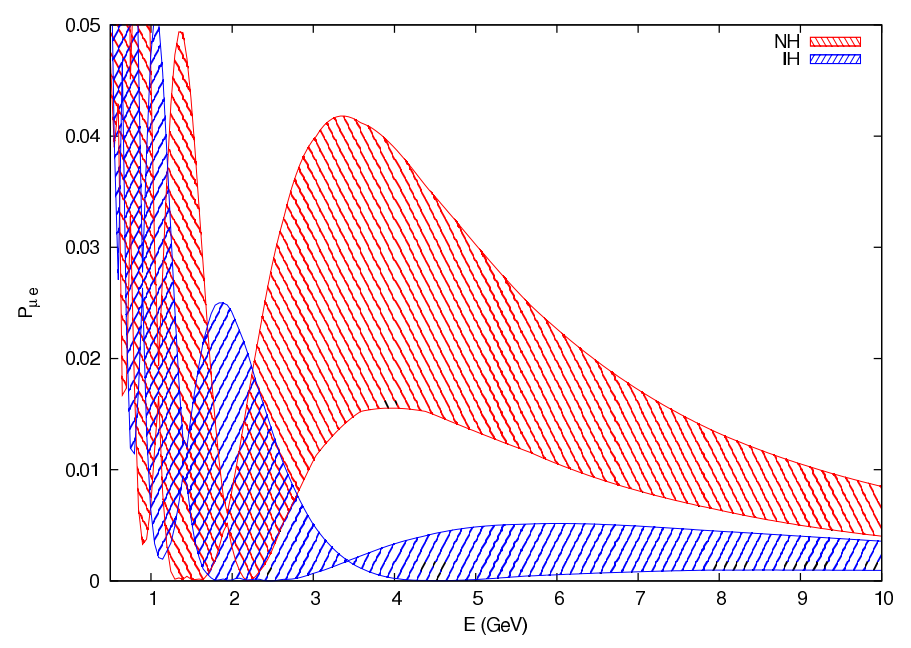

FIG. 3: $\mathrm{P}_{\mu \mathrm{e}}$ as a function of $E$ for $L=2540 \mathrm{Km}$ and $\sin ^{2} 2 \theta_{13}=0.02 . \mathrm{P}_{\mu \mathrm{e}}$ is plotted for both $\mathrm{NH}$ and IH, each for the full range of values of $\delta_{C P}$.

separation between $\mathrm{P}_{\mu \mathrm{e}}(N H)$ and $\mathrm{P}_{\mu \mathrm{e}}(I H)$ as illustrated in Fig. 3. This is true for values of $\theta_{13}$ at least as small as those detectable by the current reactor experiments. In addition, $\mathrm{P}_{\mu \mathrm{e}}(N H)$ retains its dependence on $\delta_{C P}$ unlike in the magic baseline case. This, in turn, can be helpful in measuring $\delta_{C P}[30]$.

In our calculations, we have considered a superbeam experiment with a baseline of 2540 $\mathrm{km}$. Note that the distance from Brookhaven Laboratory to Homestake mine [17, 18] and that from CERN to Pyhasalmi [31] are close to our shorter 'magical' baseline. Since our energy range of interest is around $3-4 \mathrm{GeV}$, the neutrino source must have unoscillated event spectrum peaking in this energy range. The NuMI beam in the medium energy option has an unoscillated event spectrum that peaks at $3.5 \mathrm{GeV}$ for locations $7 \mathrm{mr}$ off the beam axis [8].

\section{RESULTS}

We have done all our calculations using the software package GLoBES [32, 33]. The source is taken to be a NuMI like beam in the medium energy configuration. The detector is assumed to be a 100 kton totally active scintillator detector whose capabilities are similar to $\mathrm{NO} \nu \mathrm{A}$. Such a detector is placed at a distance $2540 \mathrm{~km}$ from the source at a $7 \mathrm{mr}$ off-axis location. The fluxes for this location were calculated using the program from [34]. The beam 
power is taken to be such that it corresponds to $10 \times 10^{20} \mathrm{POT} / \mathrm{yr}$. This is about $30 \%$ more than the design power for $\mathrm{NO} \nu \mathrm{A}$. Thus we have an exposure of $1000 \times 10^{20} \mathrm{kton}-\mathrm{POT} / \mathrm{yr}$. This is about 10 times larger than the exposure for $\mathrm{NO} \nu \mathrm{A}$. The baseline in our proposal is thrice as long as that of $\mathrm{NO} \nu \mathrm{A}$. Hence the factor 10 increase in exposure makes the data to have the same statistical weight as that of $\mathrm{NO} \nu \mathrm{A}$.

We have computed the $\nu_{e}$ appearance spectrum in the detector for the energy range from $250 \mathrm{MeV}$ to $10 \mathrm{GeV}$, in bins of $250 \mathrm{MeV}$ width. A Gaussian energy smearing function with a width of $0.1 \sqrt{E(G e V)}$ has been assumed for these events. We have considered four different types of events in our analysis: signal $\left(\nu_{\mu} \rightarrow \nu_{e}\right)$ events, beam background events (that is, events caused by intrinsic $\nu_{e}$ component of the beam), background events due to misidentified muons and the background events due to neutral current interactions. Energy independent cuts identical to those for $\mathrm{NO} \nu \mathrm{A}$ have been imposed on these channels, which substantially enhance the signal to background ratio [8, 35].

In our calculations, the solar parameters $\sin ^{2} \theta_{12}=0.304$ and $\Delta_{21}=7.65 \times 10^{-5} \mathrm{eV}^{2}$ have been kept fixed throughout [26, 36, 37]. The values of $\Delta_{31}$ and $\theta_{23}$ are taken from three flavour oscillation fit of the atmospheric [38] and MINOS data [39]. We take the true value of $\sin ^{2} 2 \theta_{23}$ to be its best fit value 1 . We assumed an error of $2 \%$ on this measurement. Hence the test values of $\theta_{23}$ are to be chosen to satisfy the constraint $0.94 \leq \sin ^{2} 2 \theta_{23} \leq 1$. Thus we have the range $38^{\circ} \leq \theta_{23} \leq 52^{\circ}$. The situation regarding $\Delta_{31}$ is a bit more complicated. The most accurate measurement of the larger mass-squared difference is given by the MINOS experiment [39]. If the results of MINOS are interpreted in terms of three flavour oscillations, the effective mass-squared difference measured by it is not $\left|\Delta_{31}\right|$ but is the magnitude of a linear combination of $\Delta_{31}$ and $\Delta_{21}$ [40, 41]. It is shown [41] that the linear combination is of the form $\Delta_{31}+\Delta_{21} f\left(\theta_{i j}, \delta_{C P}\right)$, where

$$
f\left(\theta_{i j}, \delta_{C P}\right)=\cos ^{2} \theta_{12}-\cos \delta_{C P} \sin \theta_{13} \sin 2 \theta_{12} \tan \theta_{23}
$$

In computing $\mathrm{P}_{\mu \mathrm{e}}(\mathrm{NH})$ and $\mathrm{P}_{\mu \mathrm{e}}(\mathrm{IH})$ we must relate the corresponding values of $\Delta_{31}$ via

$$
\Delta_{31}(\mathrm{IH})=-\Delta_{31}(\mathrm{NH})-2 \Delta_{21} f\left(\theta_{i j}, \delta_{C P}\right),
$$

rather than as $\Delta_{31}(\mathrm{IH})=-\Delta_{31}(\mathrm{NH})$. If we were to take $\Delta_{31}(\mathrm{IH})=-\Delta_{31}(\mathrm{NH})$, then we would get a significant fake hierarchy sensitivity in the $\nu_{\mu}$ disappearance channel, even for very small values of $\theta_{13}$. In our calculations, we have related $\Delta_{31}(\mathrm{NH})$ and $\Delta_{31}(\mathrm{IH})$ via 
Eq.(6) , and used the best fit value $\Delta_{31}(\mathrm{NH})=2.4 \times 10^{-3} \mathrm{eV}^{2}$, with an error of $5 \%$. The systematic errors include a 5\% normalization error and $2.5 \%$ tilt error on both the signal and the background. We have also taken into consideration a $5 \%$ error in the density profile of the earth.

First we computed the hierarchy sensitivity of this setup, running it in the neutrino mode only. In this part of the calculation, we limited ourselves to the range $0.01 \leq \sin ^{2} 2 \theta_{13} \leq 0.1$. That is, we assumed that this parameter will be determined by one of the current experiments. First we assumed NH to be the true hierarchy and computed the $\chi^{2}$ for distinguishing this from $\mathrm{IH}$, as a function of $\sin ^{2} 2 \theta_{13}$. In computing the $\chi^{2}$, we combined the results of our proposed set up with those of the reactor experiments and of $\mathrm{T} 2 \mathrm{~K}$ and $\mathrm{NO} \nu \mathrm{A}$ [7, 8, 35, 42 44]. The data from the reactor experiments provide a 'prior' on the values of $\sin ^{2} 2 \theta_{13}$. In computing $\chi^{2}$, we have marginalized over the range $0.01 \leq \sin ^{2} 2 \theta_{13} \leq 0.1$. We have varied the $\mathrm{CP}$ phase over the full range $-\pi \leq \delta_{C P} \leq \pi$ in computing the event rates both for $\mathrm{NH}$ and for $\mathrm{IH}$. We have then repeated the above calculation, this time assuming IH to be the true hierarchy. The results of these calculations are tabulated in Table I. We indicate here the exposure time (in years) required for hierarchy discrimination up to a $3 \sigma$ confidence level as a function of $\sin ^{2} 2 \theta_{13}$.

From the results of the table, it is worth remarking that, the present 15 kton $\mathrm{NO} \nu \mathrm{A}$ detector, with its current design luminosity, can determine the neutrino mass hierarchy, independently of $\delta_{C P}$, in a five year neutrino run, if $\sin ^{2} 2 \theta_{13} \geq 0.03$, were the detector placed at a distance of $2540 \mathrm{~km}$ with an appropriately designed beamline.

Next, we have calculated the ability of our setup (in conjunction with T2K, $\mathrm{NO} \nu \mathrm{A}$ and the reactor experiments) to determine $\delta_{C P}$ as a function of $\sin ^{2} 2 \theta_{13}$. In addition to the data from a five year neutrino run, we assume data from a five year anti-neutrino run. We have chosen fifteen different pairs of true values of $\sin ^{2} 2 \theta_{13}$ and $\delta_{C P}$ given by $\sin ^{2} 2 \theta_{13}=0.01,0.05,0.10$ and $\delta_{C P}=-160^{\circ},-80^{\circ}, 0^{\circ}, 80^{\circ}, 160^{\circ}$. We assumed normal hierarchy and computed the allowed $1 \sigma$ and $2 \sigma$ regions in the $\sin ^{2} 2 \theta_{13}-\delta_{C P}$ plane. In Figure 4 , we have plotted the outer contours of these allowed regions, corresponding to the fifteen different true parameter sets. The panel on the left gives the results including the systematic errors, whereas the panel on the right shows the allowed contours without the systematic errors. From the right panel, we see that the allowed regions are very close to the true values and the value of 


\begin{tabular}{|c|c|c|}
\hline $\sin ^{2} 2 \theta_{13}$ (true) & Exposure time(NH) & Exposure time(IH) \\
\hline 0.10 & 0.022 & 0.048 \\
\hline 0.09 & 0.026 & 0.057 \\
\hline 0.08 & 0.031 & 0.068 \\
\hline 0.07 & 0.040 & 0.082 \\
\hline 0.06 & 0.051 & 0.105 \\
\hline 0.05 & 0.070 & 0.137 \\
\hline 0.04 & 0.104 & 0.195 \\
\hline 0.03 & 0.180 & 0.420 \\
\hline 0.02 & 0.425 & 2.600 \\
\hline 0.01 & 2.950 & 4.800 \\
\hline
\end{tabular}

TABLE I: Exposure time in years required for $3 \sigma$ hierarchy discrimination, for $1000 \times 10^{20}$ kton$\mathrm{POT} / \mathrm{yr}$. The second (third) column shows the results if $\mathrm{NH}$ (IH) is the true hierarchy. It is assumed that the experiment runs in neutrino mode only.

$\delta_{C P}$ can be determined with an uncertainty of about $10^{\circ}$. The value of $\sin ^{2} 2 \theta_{13}$ is rather tightly constrained by the data from reactor neutrino experiments. However, the inclusion of systematic errors makes the allowed regions considerably larger. $\sin ^{2} 2 \theta_{13}$ is allowed to deviate from its true value by about 0.005 and the uncertainty in determining $\delta_{C P}$ rises to about $20^{\circ}$.

We have also computed the physics capabilities of the proposed setup as a stand alone experiment. As mentioned before, in the previous calculations, we have taken a lower limit of $0.01 \leq \sin ^{2} 2 \theta_{13}$. The reason for this is, that for values of $\theta_{13}$ above this limit, the data from the reactor experiments provide a 'prior'. In the calculations discussed below, we did not impose this lower limit on $\theta_{13}$ but allowed it to be as small as $\sin ^{2} 2 \theta_{13} \simeq 10^{-4}$.

In computing the results discussed below, we have assumed a 5 year neutrino and a 5 year anti-neutrino run. Based on this data, we computed the sensitivity of this set up to

- measure non-zero $\theta_{13}$

- determine hierarchy 

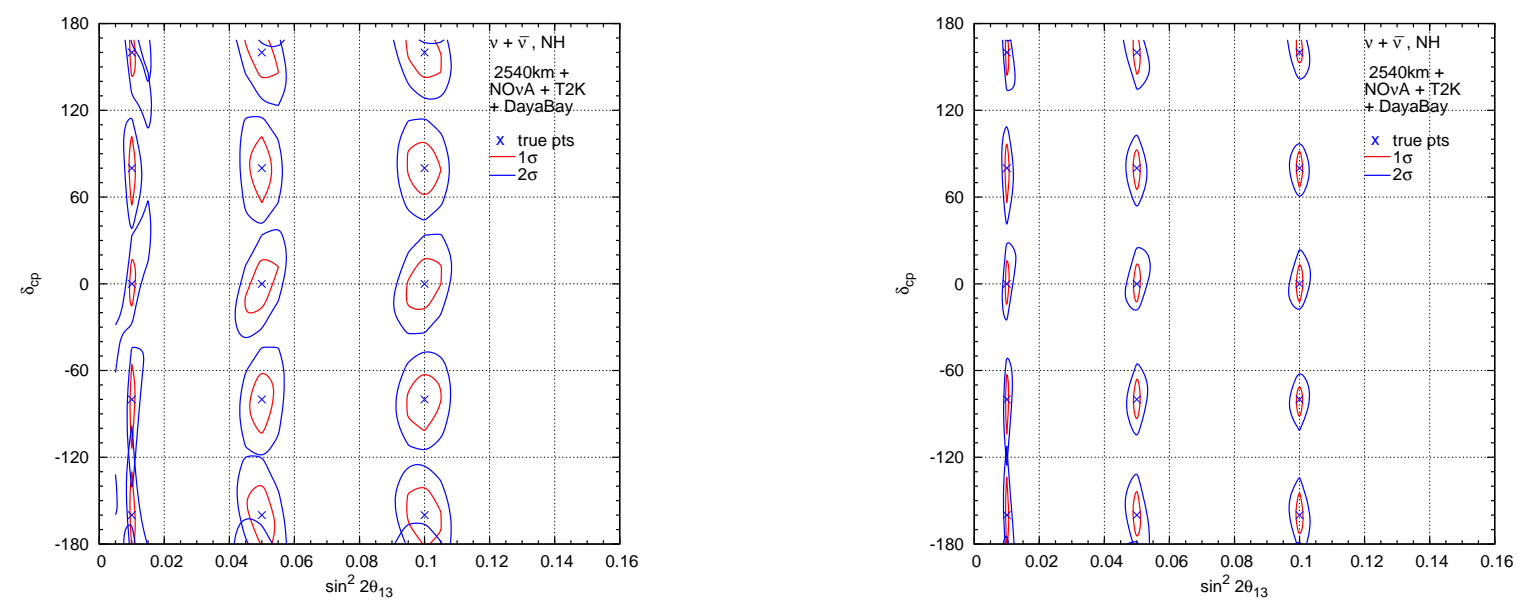

FIG. 4: $1 \sigma$ and $2 \sigma$ contours for sensitivity in the $\sin ^{2} 2 \theta_{13}-\delta_{C P}$ plane. The plot on the right is without systematics.

- rule out the CP conserving value of $\delta_{C P}=0$ or $\pi$.

In figure 5, we plot the true values of $\sin ^{2} 2 \theta_{13}$ for which this parameter can be distinguished from 0 . We do this as a function of the true value of $\delta_{C P}$ for both $\mathrm{NH}$ and IH. If $\mathrm{NH}$ is the true hierarchy, then values of $\sin ^{2} 2 \theta_{13}$ as small as $8 \times 10^{-4}$ can be measured at $3 \sigma$ level, for advantageous values of $\delta_{C P}$ (between $-\pi / 3$ to $-2 \pi / 3$ ). For adverse values of $\delta_{C P}$ (between $\pi / 6$ to $\pi / 2$ ), however, $\theta_{13}$ can be distinguished from 0 only for $\sin ^{2} 2 \theta_{13} \geq 7 \times 10^{-3}$. If $\mathrm{IH}$ is the true hierarchy, the results are similar, except for the fact that the advantageous and adverse values of $\delta_{C P}$ are exchanged.

In figure 6, we plot the sensitivity of this setup for distinguishing the mass hierarchy as a function of the true value of $\sin ^{2} 2 \theta_{13}$ and $\delta_{C P}$. Once again, for advantageous values of $\delta_{C P}$, the mass hierarchy can be determined at $3 \sigma$ for $\sin ^{2} 2 \theta_{13} \geq 8 \times 10^{-4}$, whereas for adverse values, it can be determined only for values which are about an order of magnitude larger.

Here we must insert a note of caution. Figures 5 and 6 give the sensitivity of our proposed setup as a function of the true value of $\delta_{C P}$. However, the true value of $\delta_{C P}$ is unlikely to be known from any of the current experiments. Hence, while making a statement regarding the sensitivity of our setup, we must consider the most advserse value of $\delta_{C P}$. Thus, a five year neutrino and a five year anti-neutrino run in our setup can measure, independently of $\delta_{C P}, \sin ^{2} 2 \theta_{13}$ if it is larger than $7 \times 10^{-3}$ and it can determine hierarchy for a similar lower limit on $\theta_{13}$.

Finally, figure 7 shows the ability of the setup to exclude the CP-conserving case, $\delta_{C P}=$ 
$0, \pi$. We find that the maximal phase $\delta_{C P}= \pm \pi / 2$ can be distinguished from $\delta_{C P}=0, \pi$ for $\sin ^{2} 2 \theta_{13} \geq 0.01$. But for other values, discrimination from CP conserving case is possible only for larger values of $\theta_{13}$. In fact, for $\delta_{C P}$ outside the two ranges $-2 \pi / 3$ to $-\pi / 3$ and $\pi / 3$ to $2 \pi / 3$, the discrimination from $\mathrm{CP}$ conserving case is not possible even for the largest allowed value of $\theta_{13}$.

H2B collaboration [45] have considered a neutrino superbeam experiment with a baseline in excess of $2000 \mathrm{~km}$. Their setup envisaged directing a $4 \mathrm{MW}$ neutrino superbeam, with peak flux at $E_{\nu}=4 \mathrm{GeV}$, from Tokai in Japan to a 100 kton water Cerenkov detector at Beijing, $2100 \mathrm{~km}$ away. Such a set up is close to satisfiying the shorter magic baseline conditions derived in section-II. They found that [46, 47] such a setup, running only in neutrino mode for five years, can determine mass hierarchy for all values of $\delta_{C P}$, if $\sin ^{2} 2 \theta_{13} \geq 0.02$.

There was a previous study of a superbeam proposal with $2540 \mathrm{~km}$ baseline [17, 18]. In this proposal, a wide band neutrino beam, with $0.5 \leq E_{\nu} \leq 7 \mathrm{GeV}$ energy range from Brookhaven National Laboratory is directed at a 500 kton water Cerenkov detector at Homestake mine. The beam is expected to be produced by $28 \mathrm{GeV}$ protons with $1 \mathrm{MW}$ intensity. This corresponds to $22 \times 10^{20} \mathrm{POT} / \mathrm{yr}$ assuming one year contains $10^{7}$ sec running time [48, 49]. The detector is assumed to be along the beamline, rather than at an off-axis location, which leads to the rather wide range in the energy. With a 5 year run in neutrino mode only, this setup can set a $90 \%$ confidence level upper limit $\sin ^{2} 2 \theta_{13} \leq 0.005$ if NH is the true hierarchy and $\sin ^{2} 2 \theta_{13} \leq 0.02$ if $\mathrm{IH}$ is the true hierarchy. It can also determine $\delta_{C P}$ with about a $25 \%$ uncertainty.

A few years ago, a US long baseline neutrino study group advocated a $1300 \mathrm{~km}$ baseline experiment to determine the unknown neutrino parameters from oscillations [48, 49]. They considered a wide band neutrino beam with $0.25 \leq E_{\nu} \leq 7 \mathrm{GeV}$ energy range directed from Fermilab to a 300 kton water Cerenkov detector at Homestake mine. They assumed a neutrino run with $30 \times 10^{20}$ POT with a similar anti-neutrino run. The exposure of this setup, in POT-kton, is twice that of the setup we considered. The CP-violation discovery potential of this setup is better but the hierarchy determination potential of our proposal is better [50].

Recently, there was another study which considered additional magical properties of the $2540 \mathrm{~km}$ baseline [30]. In that study, the following set up was considered. The neutrino source is a low energy neutrino factory with a flux of $5 \times 10^{21}$ muons/year with the muon 
energy of $5 \mathrm{GeV}$. The detector is a 25 kton totally active scintillator detector and it is assumed that this setup will run with positive muons for 2.5 years. With these beam and detector specifications, the authors showed that $\theta_{13}$ can be measured for values $\sin ^{2} 2 \theta_{13} \geq$ $4 \times 10^{-3}$ and the hierarchy can be determined for $\sin ^{2} 2 \theta_{13} \geq 8 \times 10^{-3}$. This set up seems to have a good ability to discover non-zero $\delta_{C P}$.

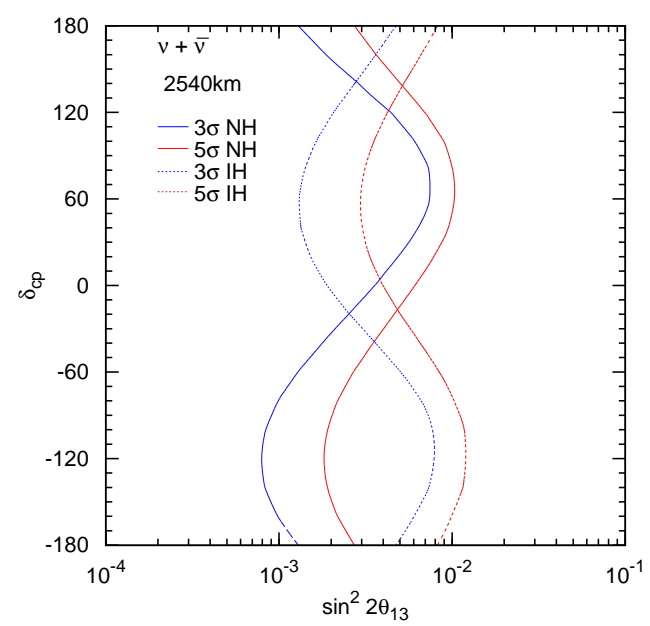

FIG. 5: $3 \sigma$ and $5 \sigma$ contours in the $\sin ^{2} 2 \theta_{13}-\delta_{C P}$ plane for excluding $\theta_{13}=0$.

\section{CONCLUSION}

In an earlier paper, we demonstrated that a neutrino superbeam experiment with a 2540 $\mathrm{km}$ baseline is particularly well suited to determine neutrino mass hierarchy, independently of the value of $\delta_{C P}$. In this paper, we performed a full-fledged study of the physics potential of such an experiment. We considered a 100 kton totally active scintillator ( $\mathrm{NO} \nu \mathrm{A}$ like) detector with the neutrino source being a NuMI-like beam in medium energy option with 


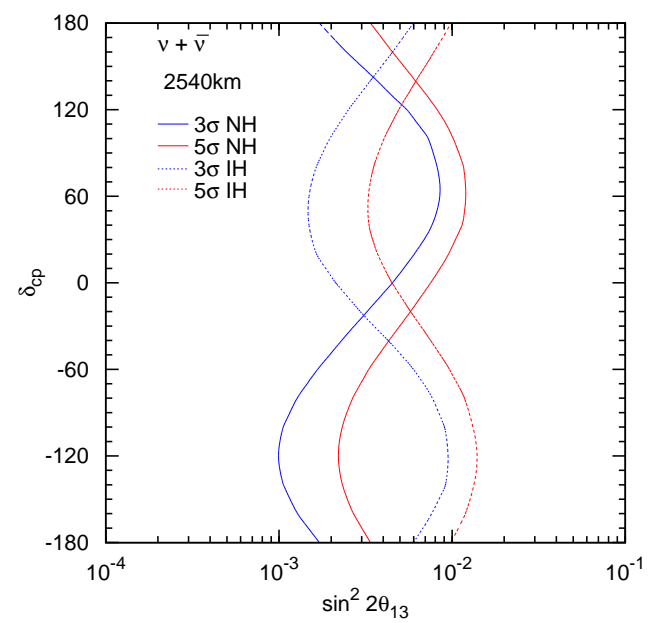

FIG. 6: $3 \sigma$ and $5 \sigma$ contours in the $\sin ^{2} 2 \theta_{13}-\delta_{C P}$ plane for excluding the 'wrong' hierarchy.

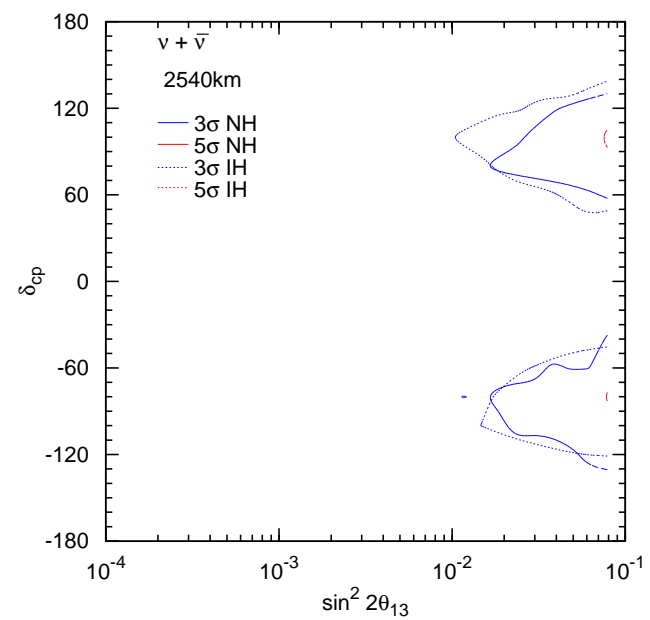

FIG. 7: $3 \sigma$ and $5 \sigma$ contours in the $\sin ^{2} 2 \theta_{13}-\delta_{C P}$ plane for excluding CP conservation.

$10 \times 10^{20} \mathrm{POT} / \mathrm{yr}$. We performed the analysis using GLoBES software, where all the appropriate background events have been included and background suppression factors relevant for $\mathrm{NO} \nu \mathrm{A}$ are imposed. We demonstrate that a five year neutrino run in this setup, together with data from reactor neutrino experiments, can determine the neutrino hierarchy independently of $\delta_{C P}$ for $\sin ^{2} 2 \theta_{13} \geq 0.01$. This statement holds irrespective of whether the true 
hierarchy is $\mathrm{NH}$ or IH. With additional data from a five year anti-neutrino run, this setup is capable of determining $\delta_{C P}$ with an uncertainty of about $20^{\circ}$.

If this setup is considered by itself, then the data from a five year neutrino run plus a five year anti-neutrino run can measure non-zero $\theta_{13}$ and determine neutrino mass hierarchy for $\sin ^{2} 2 \theta_{13} \geq 7 \times 10^{-3}$. These statements hold true for all possible values of $\delta_{C P}$. It is also possible to obtain signals for CP violation, for moderately large values of $\sin ^{2} 2 \theta_{13}$.

Acknowledgement We thank Ravi Shanker Singh for collaboration on an earlier paper where the magical properties of $2540 \mathrm{~km}$ baseline were first discussed. We thank Srubabati Goswami for numerous discussions regarding various aspects of $2540 \mathrm{~km}$ baseline and Patrick Huber for discussions on running of GLoBES.

[1] A. Osipowicz et al. (KATRIN), hep-ex/0109033.

[2] J. Wolf (KATRIN), Nucl. Instrum. Meth. A623, 442 (2010).

[3] F. T. Avignone III, S. R. Elliott and J. Engel, Rev. Mod. Phys. 80, 481 (2008).

[4] F. Ardellier et al (Double-Chooz), hep-ex/0405032; hep-ex/0606025.

[5] J. K. Ahn etl (RENO), arXiv:1003.1391.

[6] X. Guo et al (Daya Bay), hep-ex/0701029.

[7] Y. Itow et al (T2K), hep-ex/0106019.

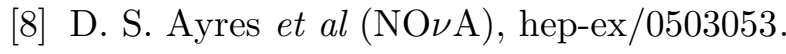

[9] P. Huber, M. Lindner, T. Schwetz and W. Winter, JHEP 0911, 044 (2009).

[10] H. Minakata and H. Nunokawa, JHEP 0110, 001 (2001).

[11] J. Burguet-Castell et al, Nucl. Phys. B608, 301 (2002).

[12] H. Minakata, H. Nunokawa and S. Parke, Phys. Rev. D 66, 093012 (2002).

[13] P. Huber, M. Maltoni and T. Schwetz, Phys. Rev. D 71, 053006 (2005).

[14] T. Kajita et al, Phys. Rev. D75, 013006 (2007).

[15] K. Hagiwara, N. Okamura, K.-i. Senda, Phys. Lett. B 637, 266 (2006), B 641,486 (2006) (E).

[16] O. Mena, S. Palomares-Ruiz and S. Pascoli, Phys. Rev. D 72, 053002 (2005); Phys. Rev. D 73, 073007 (2006).

[17] M. Diwan et al, Phys. Rev. D 68, 012002 (2003).

[18] M. Diwan et al, Report of the BNL Neutrino Working Group, hep-ex/0211001. 
[19] M. Aoki, K. Hagiwara and N. Okamura, Phys. Lett. B554, 121 (2003).

[20] V. Barger, D. Marfatia and K. Whisnant, Phys. Rev. D 65, 073023 (2002).

[21] P. Huber and W. Winter, Phys. Rev. D 68037301 (2003).

[22] S. K. Raut, R. S. Singh and S. Uma Sankar, Phys. Lett. B 696, 227 (2011).

[23] A. Cervera et al, Nucl. Phys. B579, 17 (2001); B593 731 (2001) (E).

[24] M. Freund, Phys. Rev. D 64, 053003 (2001).

[25] E. K. Akhmedov, R. Johansson, M. Lindner, T. Ohlsson and T. Schwetz, JHEP 0404, 078 (2004).

[26] Thomas Schwetz, Phys. Scripta T 127, 1 (2006).

[27] L. Wolfenstein, Phys. Rev. D 17, 2369 (1978).

[28] M. Narayan and S. Uma Sankar, Phys. Rev. D61, 013003 (2000).

[29] A. Yu. Smirnov, hep-ph/0610198.

[30] A. Dighe, S. Goswami and S. Ray, Phys. Rev. Lett. 105, 261802 (2010).

[31] J. Peltoniemi, arXiv:0911.5234

[32] P. Huber, M. Lindner and W. Winter, Comput. Phys. Commun. 167, 195 (2005).

[33] P. Huber, J. Kopp, M. Lindner, M. Rolinec and W. Winter, Comput. Phys. Commun. 177, $432(2007)$.

[34] These fluxes and interpolating programs are available at the website of $M$. Messier: http://enrico1.physics.indiana.edu/messier/off-axis/spectra/

[35] T. Yang and S. Wojcicki $(\mathrm{NO} \nu \mathrm{A})$, Study of Physics sensitivity of $\nu_{\mu}$ disappearance in a totally

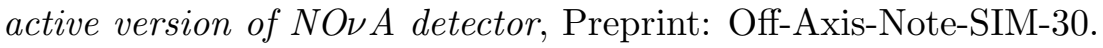

[36] K. Eguchi et al. (KamLAND), Phys. Rev. Lett. 90, 021802 (2003).

[37] T. Araki et al. (KamLAND), Phys. Rev. Lett. 94, 081801 (2005).

[38] J. Hosaka et al, (Super-Kamiokande) Phys. Rev. D74, 032002 (2006).

[39] P. Adamson et al. (MINOS), Phys. Rev. Lett. 101, 131802 (2008).

[40] A. De Gouvea, J. Jenkins and B. Kayser, Phys. Rev. D 71, 113009 (2005).

[41] H. Nunokawa, S. Parke and R. Zukanovich Funchal, Phys. Rev. D 72, 013009 (2005).

[42] P. Huber, J. Kopp, M. Lindner, M. Rolinec and W. Winter, JHEP 0605072 (2006).

[43] P. Huber, M. Lindner and W. Winter, Nucl. Phys. B 645, 3 (2002).

[44] M. Ishitsuka, T. Kajita, H. Minakata and H. Nunokawa, Phys. Rev. D 72, 033003 (2005).

[45] H. Chen et al (H2B) hep-ph/0104266. 
[46] Y. F. Wang, K. Whisnant, Z. Xiong, J. M. Yang and B.-L. Young, Phys. Rev. D65, 073021 (2002).

[47] K. Whisnant, J. M. Yang and B.-L. Young, Phys. Rev. D67, 013004 (2003).

[48] V. Barger et al, Phys. Rev. D74, 073004 (2006).

[49] V. Barger et al, Report of the US long baseline neutrino experiment study, arXiv:0705.4396.

[50] P. Huber and J. Kopp, JHEP 1103013 (2011); 1105024 (2011) (E). 